\title{
A standardised approach to pre-hospital RSI in the UK; utility, governance and content of current pre-induction checklists
}

\author{
Mark R Burgess ${ }^{1 *}$, Zane Perkins ${ }^{2}$ \\ From London Trauma Conference 2014 \\ London, UK. 9-12 December 2014
}

\section{Background}

Pre-hospital Rapid Sequence Induction (RSI) is often performed on patients nearing physiological exhaustion in a complex, challenging environment [1]. Standard Operating Procedures (SOPs) and checklists can be used to improve patient safety [2]. The UK incidence of pre-hospital RSI and the utility and content of these safety constructs are unknown.

\section{Methods}

A piloted survey was sent to the lead clinicians for all UK pre-hospital services with potential to be able to deliver RSI. Data was compared for high volume ( $>50$ RSIs per annum) and low volume ( $\leq 50$ RSIs per annum). Another piloted survey was sent to UK clinicians who themselves perform pre-hospital RSI. Current pre-induction checklists were compared and contrasted in terms of length, content and format.

\section{Results}

58 individual services were identified with $76.8 \%$ responding. $69.8 \%$ of services have RSI capabilities, $26.7 \%$ of which throughout a 24-hour period. 1564 RSIs are performed per annum.

SOPs for RSI are used by $80 \%$ and checklists by $76.8 \%$ of services, ( $>$ commonly in high volume services). $40 \%$ of these teams have a separate 'crash-induction' checklist. Review and revision of checklist content with involvement of clinicians is more common in high volume Vs. low volume services. The majority of all clinicians surveyed responded that they both prefer a standardized approach to RSI and that it is safer than allowing absolute autonomy.

There was a large variation in length, content, style and format between the checklists analysed.

\section{Discussion}

Despite the availability of pre-hospital RSI being sporadic, it is performed commonly in the UK. SOPs and safety checklists are used more commonly by high volume teams. In the challenging setting of pre-hospital care, these safety constructs may liberate spare bandwidth for utilization on other tasks, although care must be taken to limit the length and simplify the language as much as possible.

\section{Authors' details}

${ }^{1}$ Department of Anaesthetics, Royal Cornwall Hospital, Cornwall, UK. ${ }^{2}$ Centre for Trauma Science, Queen Mary University of London, UK.

Published: 11 September 2015

\section{References}

1. Perkins ZB, Wittenberg MD, Nevin D, et al: The relationship between head injury severity and haemodynamic response to tracheal intubation. The Journal of Trauma 2013, 74(4):1074-1080.

2. Rognas L, Hansen TM, et al: Standard operating procedure changed prehospital critical care anaesthesiologists' behaviour: a quality control study. Scand J Trauma Resusc Emerg Med 2013, 21:84.

\section{doi:10.1186/1757-7241-23-S2-A16}

Cite this article as: Burgess and Perkins: A standardised approach to pre-hospital RSI in the UK; utility, governance and content of current pre-induction checklists. Scandinavian Journal of Trauma, Resuscitation and Emergency Medicine 2015 23(Suppl 2):A16.

* Correspondence: mda05mtb@gmail.com

'Department of Anaesthetics, Royal Cornwall Hospital, Cornwall, UK

Full list of author information is available at the end of the article 